\title{
Oclusão bilateral balanceada - um princípio básico de prótese total. Relato de caso
}

\author{
Balanced bilateral occlusion- a basic principle of full arch prosthesis. Case report \\ Oclusión bilateral balanceada - un principio básico de prótesis total. Reporte de caso clínico \\ Jadison Junio CONFORTE 1 \\ Thiago MACHADO2 \\ Rodrigo Capalbo da SILVA2 \\ Henrique HADAD ${ }^{2}$ \\ Francisley Ávila SOUZA2 \\ Wirley Gonçalves ASSUNÇÃO' ${ }^{1}$

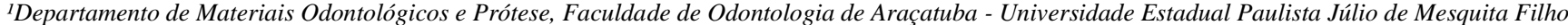 \\ (UNESP) 16015-050 Araçatuba-SP, Brasil

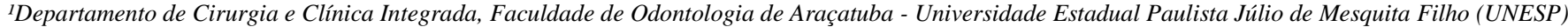 \\ 16015-050 Araçatuba-SP, Brasil
}

\begin{abstract}
Resumo
A oclusão equilibrada e em perfeita harmonia é objetivo primordial em prótese total. Este trabalho tem como objetivo mostrar a influência da utilização dos princípios na confecção de prótese total e seu resultado. Paciente do sexo feminino, 67 anos, portadora de prótese total bimaxilar buscou atendimento especializado tendo como queixa principal mobilidade de sua prótese total superior, acompanhada de queixa estética. Dentre os planos de tratamento propostos, foi realizada a confecção de novas próteses totais respeitando princípios e passos visando o reestabelecimento de função e estética. No momento da instalação observou-se a oclusão bilateral balanceada, tendo objetivo do tratamento alcançado. A confecção de prótese odontológica deve respeitar os princípios básicos e fundamentais de confecção, bem como os requisitos inerentes às possibilidades reabilitadoras, tendo em vista que o seu resultado influencia diretamente na qualidade de vida do paciente.

Descritores: Oclusão Dentária; Prótese Total; Qualidade de Vida.
\end{abstract}

\section{Abstract}

The right balanced occlusion and perfect harmony are the main target in full arch prosthesis. The aim of this work is to evidence the influence on correct use of full arch prosthetic rehabilitation precepts in manufacture and its result. A 67-year-old female patient with a total bimaxillary prosthesis sought specialized care having as main complaint the mobility of her maxillary total prosthesis and aesthetic complaint. Among the proposed treatment plans, it was carried out making new dentures respecting principles and steps towards the function and aesthetics re-establishment. When installing observed balanced bilateral occlusion, with the aim reached treatment. The preparation of dental prosthesis must respect the steps and basic principles of preparation and requirements inherent to rehabilitation possibilities, considering that its result directly influences the quality of life of patient.

Descriptors: Dental Occlusion; Denture, Complete; Quality of Life

\section{Resumen}

La oclusión equilibrada y en perfecta armonía eres objetivo primordial en prótesis total. Este trabajo tiene como objetivo mostrar la influencia de la utilización de los básicos principios en la confección de prótesis total y su resultado. Paciente, sexo femenino, 67 años, hace uso de prótesis total bimaxilar recurrió al atendimiento especializado y como principal reclamación la movilidad de su prótesis total superior tan cómo la estética. Entre las proposiciones de planos de tratamiento, se fue realizada la confección de nuevo par de prótesis totales respetando los principios y etapas en busca de la devolución de función y estética. Al momento de la instalación se fue posible observar la oclusión bilateral balanceada, teniendo el objetivo alcanzado. Para que la prótesis odontológica sea confeccionada se debe respetar los principios básicos fundamentales de la confección y los requisitos inherentes a las posibilidades rehabilitadoras, observando que su resultado tiene directa influencia en la calidad de vida del paciente.

Descriptores: Oclusión Dental; Dentadura Completa; Calidad de Vida

\section{INTRODUÇÃO}

Para a reabilitação do paciente desdentado total em um ou ambos os arcos com prótese total a oclusão será bilateral balanceada. Christensen ${ }^{1}$ em 1905 foi pioneiro para o estudo da oclusão bilateral balanceada em prótese total, onde, posteriormente, outros autores vieram a seguir tal padrão oclusal em prótese total removível, sendo atualmente preconizado para estes $\operatorname{casos}^{2,3}$.

A oclusão equilibrada e em perfeita harmonia é objetivo primordial em prótese total ${ }^{4}$. Os planos das bases de prova serão parâmetros de referência para estabelecer aspectos estéticos e funcionais do paciente, através dos planos de cera das bases de prova que a orientação em paralelo ao plano de Camper deverá ser procedida ${ }^{5}$. O adequado registro da dimensão vertical de oclusão, de forma que toda a extensão dos planos esteja em contato, facilitarão a montagem de dentes em oclusão bilateral ${ }^{6}$.
Assim sendo, o respeito à técnica de confecção de uma prótese total, seguindo todas as etapas necessárias, permite o restabelecimento da função e estética facial do paciente de forma satisfatória e efetiva.

\section{CASO CLÍNICO}

Paciente do sexo feminino, 67 anos de idade, leucoderma, portadora de prótese total bimaxilar buscou atendimento especializado queixando-se de mobilidade de sua prótese total superior. A paciente foi submetida à anamnese e à oroscopia e ectoscopia não se observou nenhuma alteração digna de nota das estruturas anatômicas e tegumentar. Foi constatada a falta do contato bilateral nos dentes posteriores, e mordida aberta posterior expressiva ao lado esquerdo.

Como queixa principal relatou que ao mastigar, a prótese superior entrava em movimento a 
partir do toque dos dentes da prótese total inferior, gerando incômodo por não poder exercer tal função de maneira adequada. Ademais, os alimentos entravam no espaço criado pelas próteses, gerando desconforto e insegurança em razão do medo existente ligado à possibilidade de a prótese ser expelida da cavidade bucal quando em função. Desta forma, a paciente buscou por atendimento odontológico a buscar por maior estabilidade e retenção.

Em comprovada a falta de oclusão bilateral balanceada (Figura 1), foi então proposto à paciente as opções para correção deste problema, bem como para melhorar a sua saúde do aparelho estomatognático, das quais citam-se: $1^{\circ}$ correção oclusal para bilateral balanceada com manutenção da prótese já utilizada; $2^{\circ}$ confecção de novo par de próteses totais seguindo os preceitos para uma prótese total adequada; $3^{\circ}$ novo par de próteses do tipo protocolo de Bränemark.

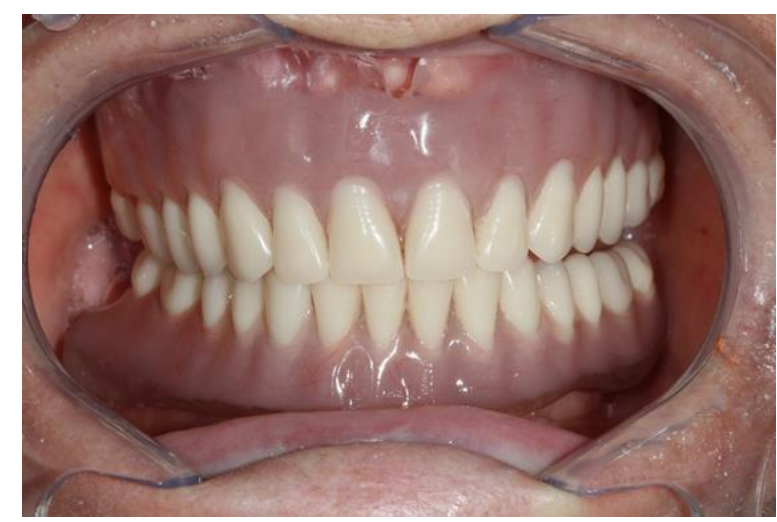

Figura 1: Prótese total bimaxilar sem oclusão bilateral balaceada.

Dos planos de tratamento propostos, a paciente optou pela confecção de um par de próteses totais nova, já que ela também se queixava da estética de sua prótese.

Assim sendo, seguiu-se a confecção das próteses totais, superior e inferior, respeitando os devidos princípios e passos para sua confecção. Ao momento da instalação, o correto ajuste oclusal efetuado possibilitou oclusão bilateral balanceada com correção das falhas observadas em próteses anteriores (Figura 2).

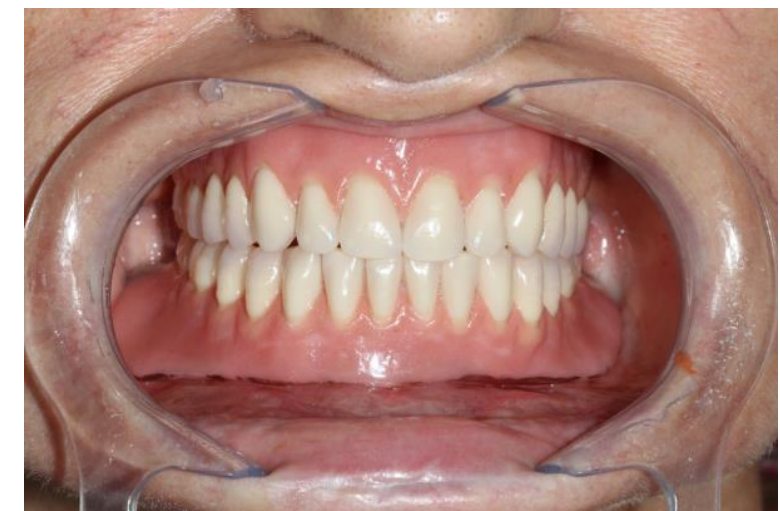

Figura 2: Prótese total bimaxilar com oclusão bilateral balaceada.
No acompanhamento de dois meses a paciente relatou melhora da estabilidade e retenção das próteses quando comparada as antigas, influenciando diretamente em sua qualidade de vida.

\section{DISCUSSÃO}

A prótese total visa auxiliar a recomposição artificial as estruturas perdidas do aparelho estomatognático 5 .

A correta orientação dos planos oclusal e altura da dimensão vertical de oclusão permite a devolução das funções perdidas em indivíduos reabilitados com próteses totais ${ }^{7}$. A harmonia da oclusão em prótese total é de grande importância para o aparelho estomatognático. Onde a oclusão bilateral equilibrada e harmônica compreende o contato dos dentes tanto do lado de trabalho, bem como o de balanceio. Quando há desarmonia oclusal ocorre perda do desempenho mastigatório ${ }^{8}$. A oclusão bilateral balanceada é necessária para a estabilidade funcional das próteses totais, bem como importante no fornecimento de melhor eficiência mastigatória ${ }^{9-11}$.

A prótese total sem a adequada oclusão bilateral balanceada gera instabilidade da prótese. Uma vez restabelecida a oclusão balanceada das próteses haverá pouca movimentação ${ }^{12}$. Compagnoni et al. ${ }^{13}$ relatam que a desoclusão lateral não alterou na função mastigatória desempenhada pelo paciente ${ }^{13}$. A eficiência mastigatória e força máxima da oclusão sofrem influência dos esquemas oclusais em prótese total $^{14}$, já que há um aumento do número de ciclos mastigatórios e prolongamento do tempo de mastigação nos pacientes com prótese totais convencionais ${ }^{15}$.

A correta orientação dos planos de cera, com os contatos múltiplos e simultâneos e bilaterais auxilia na montagem dos dentes em cera. Normalmente isso é perdido após a acrilização ${ }^{16}$.

A oclusão bilateral balanceada assume um papel fisiológico importante na reabsorção do rebordo residual em pacientes usuários de prótese total, pois esta oclusão preserva o rebordo residual e influencia a estabilidade das próteses ${ }^{17}$.

Tão logo, manter a oclusão bilateral em prótese total trará estabilidade, retenção protética, melhora da eficiência mastigatória, menor reabsorção do rebordo residual, qualidade de vida ao paciente.

\section{CONSIDERAÇÕES FINAIS}

A confecção de qualquer tipo de prótese odontológica deve respeitar os seus princípios de confecção e requisitos inerente às possibilidades reabilitadoras. Não devendo diferir em prótese total, haja vista que estas próteses ao entrarem em função, espera-se que as necessidades funcionais do paciente sejam atendidas e se apresentem estáveis. Este resultado é acompanhado da mudança de autoestima 
do paciente, bem como de sua vida social e profissional, implicando em melhor qualidade de vida ao mesmo.

\section{REFERÊNCIAS}

1. Christensen C. The problem of the bite. Dent Cosmos. 1905;47(10):1184-95.

2. Gomes MA, Tamaki ST, Tamaki T. Contatos de trabalho e balanceio em prótese total. Rev Odontol Univ São Paulo. 1990;4(1):49-54.

3. Turano JC, Turano LM. Fundamentos de prótese total. 3.ed. Rio de Janeiro: Quitessence; 1993.

4. Jorge JH, Varjão FM, Nogueira SS. Oclusão lingualizada para próteses totais. RGO. 2003;51(2):105-11.

5. Telles DM. Prótese total: convencional e sobre implantes. 2.ed. São Paulo: Santos; 2011.

6. Carr AB, Brown DT. McCracken: Prótese Parcial Removível. 12.ed. Rio de Janeiro: Elsevier, 2012.

7. Breda RA, Sicoli EA, Mendonça MJ, Pezzini RP. Prótese total pela técnica da zona neutra. RGO. 2006;54(3):274-79.

8. Tamaki ST, Gomes MAO, Tamaki T. Número de contatos em oclusão e articulação em prótese total. Rev Bras Odontol. 1991;48(1):40-6.

9. Georgetti MP, Georgetti BA, Corrêa GA, Magalhães Filho O. Aspectos fundamentais para a estabilidade das próteses totais. Rev Odontol Univ Santo Amaro. 2000;5(2):71-5.

10.Ruffino AR. Improved occlusion anatomy of acrylic resin denture teeth. J Prosthet Dent. 1984; 52(2):300-2.

11.Turano LM, Turano JC. Fundamentos de prótese total. 6. ed. São Paulo: Santos; 1998.

12.Dubojska AM, White GE, Pasiek S. The importance of occlusal balance in the control of complete dentures. Quintessence Int. 1998; 29(6):389-94

13.Compagnoni MA, Leles CR, Barbosa DB, Valverde GB. Oclusão em dentaduras completas. Estudo comparativo entre oclusão balanceada bilateral e desoclusão pelo canino. Rev CROMG. 2002;8(2):92-7.

14. Niwatcharoenchaikul W, Tumrasvin W, Arksornnukit M. Effect of complete denture occlusal schemer on masticatory performance and maximum occlusal force. $\mathrm{J}$ Prosthet Dent. 2014;112(6):1337-42.

15.Frota PL, Bastos PL, de Figueiredo VMG. Padrão de oclusão em prótese total: um dilema da reabilitação oral. Rev Bahiana Odonto. 2016;7(1):58-62.

16.Pomilio A, Campos Júnior WM, Tedesco AC. Alterações dimensionais da prótese total na base e nos dentes de dentaduras inferiores. RGO. 1996; 44(2):77-9.
17. Abduo J. Occlusal schemes for complete dentures: a systematic review. Int $\mathrm{J}$ Prosthodont. 2013;26(1):26-33.

\section{CONFLITO DE INTERESSES}

Os autores declaram não haver conflitos de interesse.

\section{AUTOR PARA CORRESPONDENCIA}

\section{Henrique Hadad}

henriquehadad@gmail.com

Submetido em 22/05/2018

Aceito em 04/10/2018 